\title{
The role of 99mTC-DTPA retrobulbar SPECT in staging and follow-up of Graves' orbitopathy
}

\author{
László Galuska1, Sándor K. Barna², József Varga¹, Ildikó Garai,2, Endre V. Nagy \\ ${ }^{1}$ University of Debrecen, Hungary \\ ${ }^{2}$ ScanoMed Ltd., Debrecen, Hungary
}

[Received 3 X 2017; Accepted 20 X 2017]

\begin{abstract}
The pathogenesis of Graves' orbitopathy (GO) is not completely understood. Coexistent hyperfunction of the thyroid gland is frequent; however, GO may also coexist with hypo- or euthyrodism. The course of GO is largely independent of thyroid function, although elevated serum TSH is known to negatively interfere with GO course. GO is severe in $10 \%$ of the cases; sight threatening complications may also develop. A successful therapy of GO requires the assesment of both the severity and activity of orbital inflammation. Based on relevant studies and our own experiences, the possible management choices are reviewed here. For this purpose, we compare the clinical value of imaging techniques for detecting the activity of the disease. During the last 15 years, we used ${ }^{99 m}$ Tc-DTPA retrobulbar SPECT routinely in more than 1400 patients to facilitate the right therapeutic decision. This diagnostic utility simplified management decisions compared to previously applied alternative techniques. We recommended the routine use of ${ }^{99 \mathrm{~m} T C-D T P A}$ retrobulbar SPECT for the evaluation and follow-up of GO.
\end{abstract}

\section{KEY words: GO, ${ }^{99 m}$ Tc-DTPA, hyperthyroidism}

Nucl Med Rev 2018; 21, 1: 54-58

\section{Introduction}

Graves' orbitopathy (GO) is an autoimmune disease characterized by exophthalmos, caused by the enlargement of external eye muscles, and an increase in the volume of connective tissue in the orbital space. As part of the autoimmune process, the orbital space is infiltrated by immunocompetent cells, especially macrophages, plasma cells, lymphocytes, and mast cells. Orbital fibroblasts are thought to play a crucial role in the pathogenesis by their ability to produce strongly hydrophilic glycosaminoglycans, resulting in fluid retention and edematous swelling of the orbital tissue. It is important to note that the immunological activity and clinical severity of GO are following separate courses, and activity is the primary determinant of the response to immunosuppressive treatment $[1,2]$. In an early stage of disease, marked immune activity may be accompanied by only moderate clinical signs, while later severe clinical presentation may equally be present with or without immune activity, as shown by Rundle as early as in 1960 [3]. Determining the activity of GO at each clinical assessment

Correspondence to: László Galuska, University of Debrecen, Nagyerdei krt 98, 4032, Debrecen, Hungary, e-mail: galuska.laszlo@med.unideb.hu is fundamental in the planning of the appropriate management. Immunomodulatory therapies can only be effective while there is active inflammation. On the other hand, surgery involving the external eye muscles for the correction of diplopia should only be undertaken when GO is inactive, and there is no further chance of change in muscle function due to fluctuating immune activity. The major and easiest approach to assess the activity is the estimation of the clinical activity score (CAS), first described by Mourits in 1997 [4]. However, CAS is also influenced by anterior segment changes, and easily over- or underestimates disease activity (Nagy et al.) [5]. Up till now, no reliable serum factors have been identified which could estimate immune activity in GO. For long, a highly reproducible and quantifiable method for disease activity estimation has been missing [6]. In the present review, we summarize the multidisciplinary approach to the activity and severity of GO, proposing a diagnostic strategy.

\section{Current diagnostic methods}

\section{Approach of Clinical Endocrinology}

The hormonal investigation and follow-up of patients with GO are in the traditional scope of endocrinology. The thyroid hormonal status and measurements of antibody concentrations belong here. Usually higher TSH receptor antibody levels accompany active orbital inflammation; however, low antibody levels do not exclude active GO. During the treatment of Graves' hyperthyroidism, 
overtreatment resulting in high serum TSH should be avoided, as it may negatively interfere with the course of GO [7]

\section{Ophthalmology}

The patients suffering from GO usually turn to, or are referred to an ophthalmologist. The measurement and scoring of visual symptoms and signs remain essential for patient management. While the NOSPECT classification [8] describes disease severity, for disease activity, the clinical activity score (CAS) is the gold standard. However, there is little or no correlation between the severity and activity of GO. Eye movement is assessed by the Hess chart. Proptosis is measured by Hertel ophthalmometer. The main limiting factor of any ophthalmological scoring system is that it is based on findings on the anterior orbital segment; as the immune activity is hidden in the orbit, only indirect information can be obtained about the immune process.

\section{Non-nuclear imaging}

In $\mathrm{GO}$, it is essential to check the anatomy of the intraorbital space to assess the cause and severity of exophthalmus. MRI is suitable for showing the fat and orbital connective tissue, and can describe the condition of the lacrimal glands and the ophthalmic nerves $[9,10]$. Prolongation of the T2 relaxation time in the eye muscles is a reliable MRI marker of disease activity in the muscles. However, other components of the orbital space, including fatty connective tissue, are often not correctly graded for inflammation by MRI. "A" mode ultrasonography of the eye muscles may be useful for detecting disease activity in the muscles and monitoring the changes; however, limited eye movement limits the angles of the ultrasound probe to be used for muscle diameter measurement.

\section{Methods of nuclear medicine}

Since nuclear medicine techniques can provide the functional information mentioned in the introduction that are essential for assessing disease activity, their use in clinical practice has attracted increasing attention. For a long time, in the planar gamma camera era, both the lack of GO-specific radiopharmaceuticals, and the poor spatial resolution prevented a nuclear medicine approach. First in 1999, ${ }^{111}$ In-octreotide [11-13] and labelled polyclonal human immunoglobulin [14] were tested to estimate the activity of GO. Although these methods have been accepted for the evaluation of GO activity, they are considered research approaches, as high cost remains a serious drawback. Recently, more cost-effective radioisotope methods have been introduced, with higher spatial resolution of the orbital space by SPECT. This enabled us to numerically grade the immune activity of GO. These methods meet the requirements listed above, and are becoming part of patient care in endocrinology, ophthalmology, and radiation therapy.

\section{Imaging method}

The details of DTPA orbital SPECT were described earlier [15]. Briefly, a double- or four-headed SPECT system is pre-calibrated

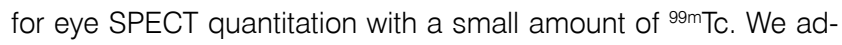
minister $7 \mathrm{MBq} / \mathrm{kg}{ }^{99 \mathrm{~m} T c-D T P A}$, recording the time of measurement for later uptake activity (UA) calculation. Twenty minutes after the intravenous injection, a four-headed SPECT (MEDISO, Budapest, Hungary) is routinely used for the acquisition of 128 projections in step-and-shoot mode over 360 degrees. For data analysis, the
DIAG and INTERVIEW software packages (MEDISO, Hungary) are used. After reconstruction and attenuation correction, we select and summarize 12 consecutive coronal, sagittal and transversal slices for visual interpretation (Figure 1).

For UA calculation, a summed transversal slice with $24.6 \mathrm{~mm}$ thickness centered on the eye-balls is used. The retrobulbar regions of interest (ROIs) are delineated, from which the activities in unit volume, expressed as proportions of the injected dose, are calculated. The upper normal UA value, based on disease-free orbits, was found to be $12 \times 10^{-6} \mathrm{ID} / \mathrm{mL}[15,16]$ (Figure 2). Occasionally orbitopathy may be one-sided (Figure 3 ).

\section{DTPA uptake values, other diagnostic methods, and treatment outcome}

In 2002 we compared DTPA SPECT data with MRI, and found good correlation regarding disease activity. It appears likely that DTPA SPECT may be comparable to octreotide and other polypeptide SPECT results. The clinical usefulness of DTPA SPECT has been proven by several clinical studies from our institution [17]. Although factors of the initiation and perpetuation of the autoimmune process in GO remain unclear [18], quantitative data of orbital inflammation in GO are essential for appropriate treatment choices [19]. In disease activity measurement and prognosis estimation, the UA has been shown a useful parameter in GO by independent authors [20]. Based on our early experience with DTPA orbital SPECT, we included it in the routine clinical evaluation of GO; we have performed more than 1400 orbital DTPA SPECTs during the last 15 years.

We tested the method for staging and follow-up of patients, in order to establish therapeutical decisions [21, 22]. In the last 10 years we use a protocol of GO patient management, which includes information obtainable by different imaging techniques. Diagnostic and therapeutic measures in GO patient management, including the DTPA orbital SPECT, are summarized in the flow chart (Figure 4).

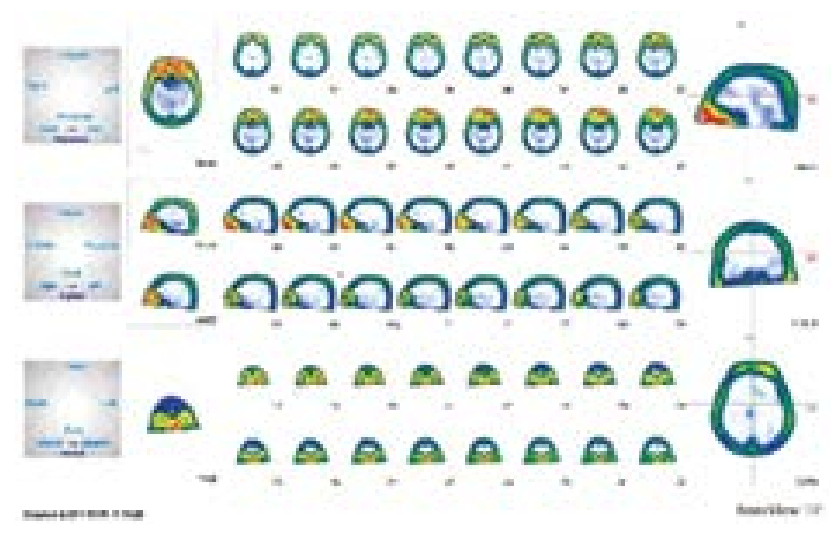

Figure 1. Sequence of $2 \mathrm{~mm}$ slices in transvers, sagittal and coronal planes after data acquisition, filtered back projection, and attenuation correction. Altogether 12 slices are summarized in which the volume of orbital space is included. Left, from up to down: sum of 34-45 transvers slices, sum of 13-24 right, 40-52 left sagittal, and 17-28 coronal slices are shown. For UA calculation we draw ROIs on selected transvers slices, producing VOls of the orbital regions (See Fig. 2). The cold area of eye globe helps the correct orientation 

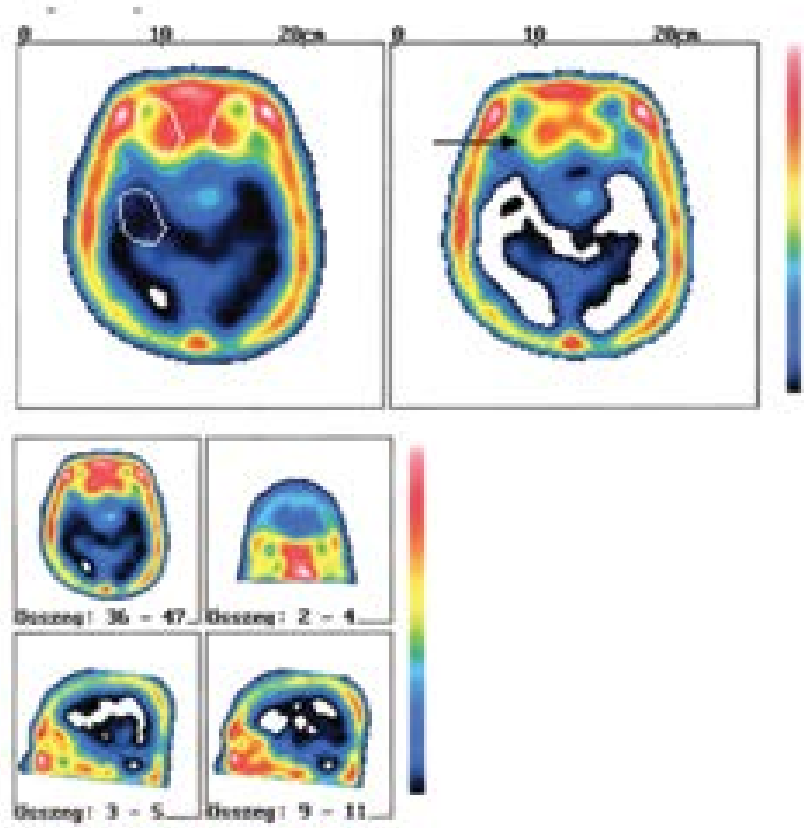

Figure 2. Typical group of 12 summarized slices from 3 , previously mentioned (Fig 1) planes, which are printed as part of the final report on a patient suffering from GO. The high DTPA uptake is visible as redcolored area (arrow) in the region of the medial eye muscle on both sides. The UA values were 13 (right) and $15 \cdot 10^{-6} \mathrm{ID} / \mathrm{mL}$ (left orbital region), showing both sided active GO
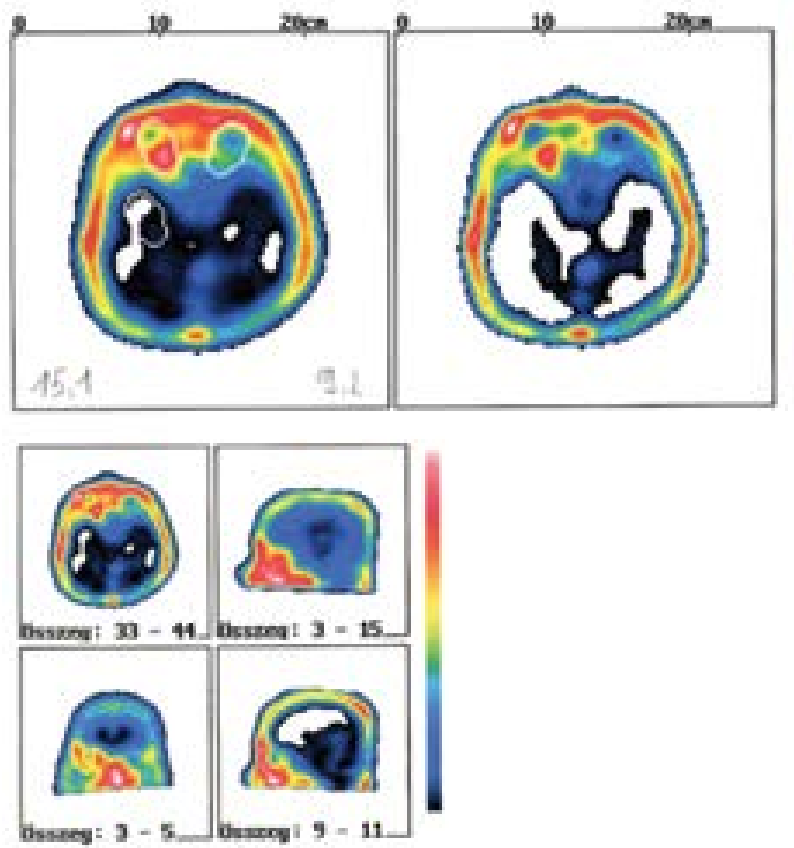

Figure 3. Asymmetric, mainly right-sided GO. The UA on the right and left side were 15.1, and 9.2, respectively

Using our flow chart in clinical routine patient care, the first specialists who most frequently meet patients with suspected GO are the ophthalmologists.

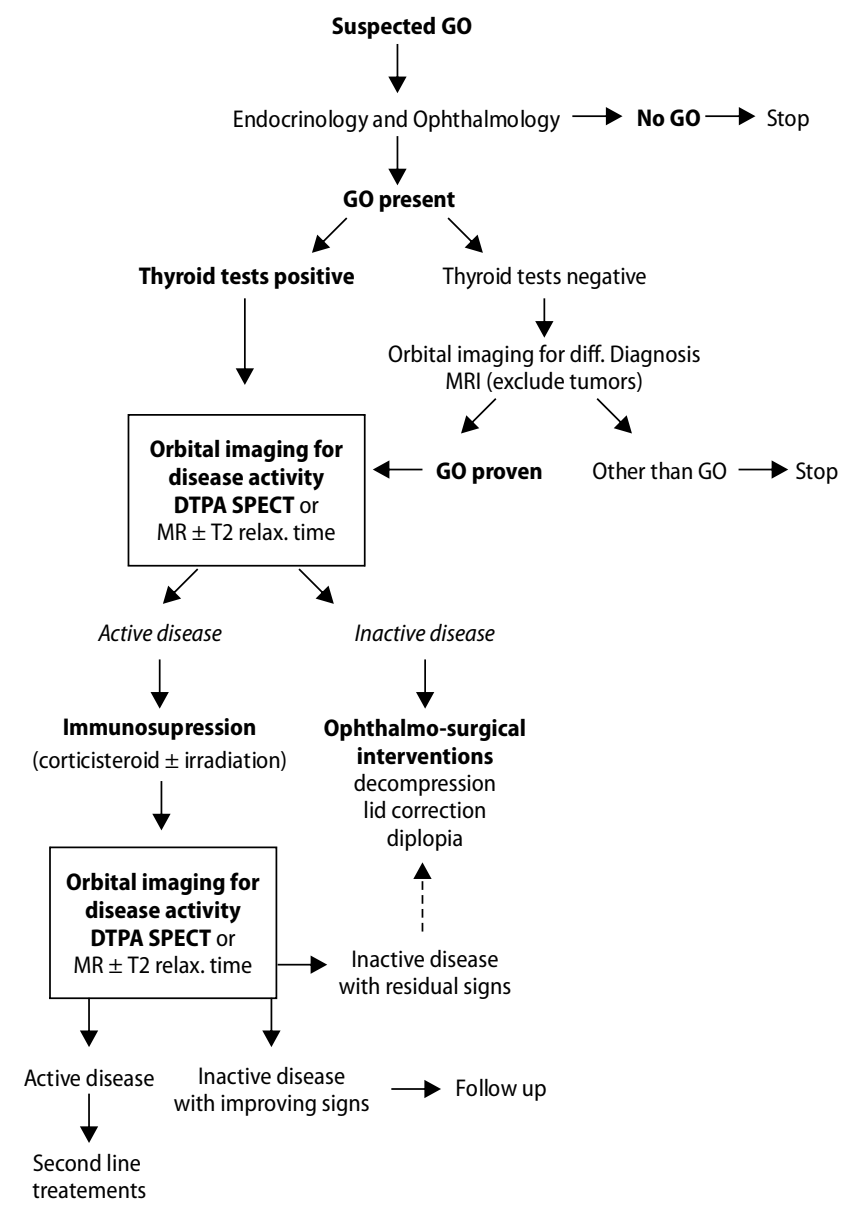

Figure 4. The suggested workup for GO. The flow chart includes imaging techniques at the major points of decision (in frames). The preferable method is SPECT if thyroid disease is present, while MR is the first choice if there are no thyroid dysfunction or thyroid antibodies (Immediate MR is required for the assessment of the optic nerve if visual acuity deteriorates any time during the course of GO)

After careful physical ophthalmological examination and the calculation of the clinical activity score (CAS), the next important diagnostic question is the biological activity of GO. MRI may exclude intraorbital tumor, and T2 relaxation time measurements in the affected muscles can help the activity estimation of GO. Sometimes the UA values are in the normal range, but the signs of inflammation are visible outside of the orbital space, in the frontal and facial sinus areas (Figure 5). In cases with symptoms of newly diagnosed or relapsed GO, and a CAS suggestive of an active GO $(C A S \geq 4)$, DTPA eye SPECT is indicated. For patients with initially normal or low DTPA uptake, steroid treatment or external irradiation had poor prognosis. In case of these patients other therapeutic measures, as decompressive surgery, fat removal (lipectomy), double vision correction, etc., should be considered (Figure 6).

\section{Conclusions}

${ }^{99 m} T c-D T P A$ is a well-known traditional radiopharmaceutical [23, 24]. More than 15 years ago we introduced DTPA orbital SPECT in the clinical management of endocrine patients suffering from 

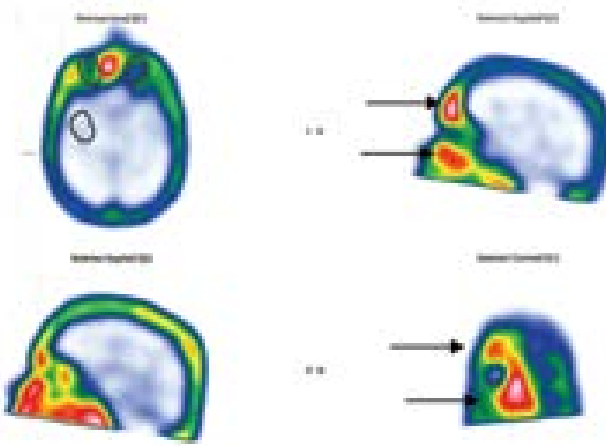

Figure 5. Figures of a patient suffering from headache. The UA values were in the normal range, but the signs of inflammation are visible in the frontal and facial sinus area (arrow)
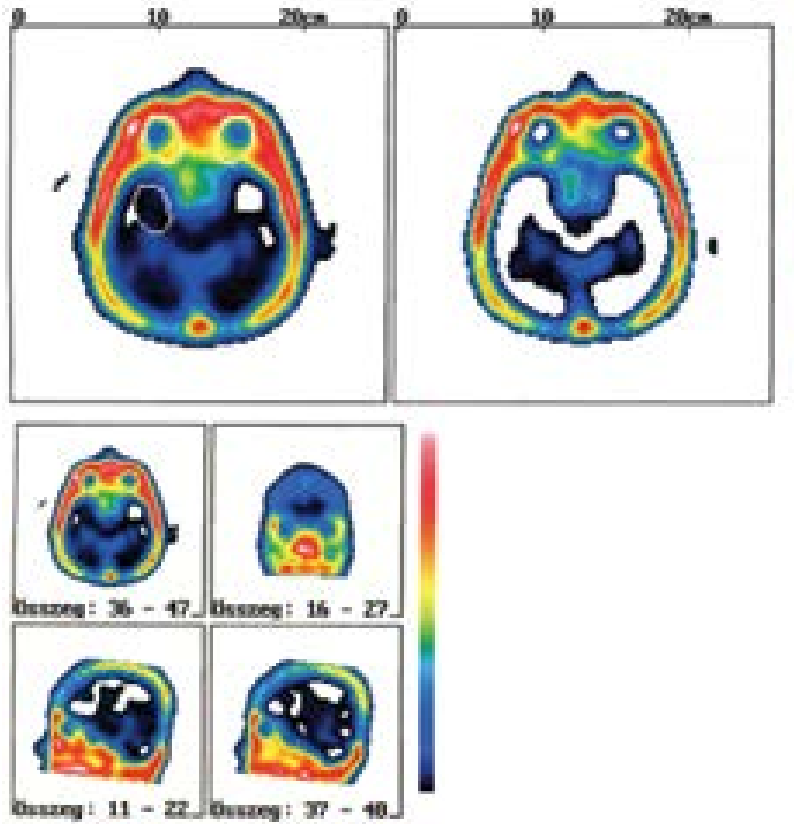

Figure 6. GO in stage of minimal inflammatory activity. The UA on the right and left side were 10.2 and 9.1 , respectively

GO. Our method of orbital UA quantification has proved to be suitable for the selection of EOP patients, providing good or poor prognosis of steroid or external irradiation therapy, as well as ophthalmologic interventions. Recently, a new software allows faster data analysis (Figure 7).

\section{References}

1. Marcocci C, Bartalena L, Bogazzi F, et al. Studies on the occurrence of ophthalmopathy in Graves' disease. Acta Endocrinol (Copenh). 1989; 120(4): 473-478, doi: 10.1530/acta.0.1200473, indexed in Pubmed: 2718699.

2. Heufelder AE. Pathogenesis of Graves' ophthalmopathy: recent controversies and progress. Eur J Endocrinol. 1995; 132(5): 532-541, doi: 10.1530/eje.0.1320532, indexed in Pubmed: 7749490.

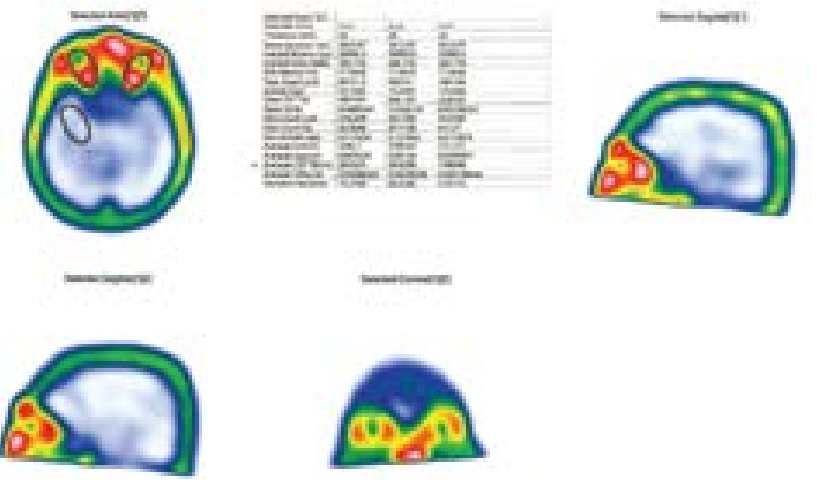

Figure 7. Both-sided active GO, documented with the new MEDISO InterViewXP software. The UA values were 18.8 and 19.1 on the right and left side, respectively. All the information is summarized in the table

3. RUNDLE FF. Management of exophthalmos and related ocular changes in Graves' disease. Metabolism. 1957; 6(1): 36-48, indexed in Pubmed: 13386967.

4. Mourits MP, Prummel MF, Wiersinga WM, et al. Clinical activity score as a guide in the management of patients with Graves' ophthalmopathy. Clin Endocrinol (Oxf). 1997; 47(1): 9-14, doi: 10.1046/j.1365-2265.1997.2331047.x, indexed in Pubmed: 9302365.

5. Nagy EV, Toth J, Kaldi I, et al. Graves' ophthalmopathy: eye muscle involvement in patients with diplopia. Eur J Endocrinol. 2000; 142(6): 591-597, doi: 10.1530/eje.0.1420591, indexed in Pubmed: 10832104.

6. Wiersinga WM, Kahaly GJ. Graves' orbitopathy A Multidisciplinary Approach Karger, Amsterdam 2007: Amsterdam.

7. Mark A, Prummel M, Wilmar M, et al. Medical management of Graves' orbitopathy. Thyroid. 1995; 5: 231-234.

8. Bartley GB, Gorman CA, Fatourechi V, et al. Diagnostic criteria for Graves' ophthalmopathy. Am J Ophthalmol. 1995; 119(6): 792-795, doi: 10.1016/s0002-9394(14)72787-4, indexed in Pubmed: 7785696.

9. Szucs-Farkas Z, Toth J, Balazs E, et al. Using morphologic parameters of extraocular muscles for diagnosis and follow-up of Graves' ophthalmopathy: diameters, areas, or volumes? AJR Am J Roentgenol. 2002; 179(4): 1005-1010, doi: 10.2214/ajr.179.4.1791005, indexed in Pubmed: 12239055.

10. Szucs-Farkas Z, Toth J, Kollar J, et al. Volume changes in intra- and extraorbital compartments in patients with Graves' ophthalmopathy: effect of smoking. Thyroid. 2005; 15(2): 146-151, doi: 10.1089/thy.2005.15.146, indexed in Pubmed: 15753674.

11. Gerding MN, van der Zant FM, van Royen EA, et al. Octreotide-scintigraphy is a disease-activity parameter in Graves' ophthalmopathy. Clin Endocrinol (Oxf). 1999; 50(3): 373-379, indexed in Pubmed: 10435064.

12. Krassas GE, Doumas A, Kaltsas T, et al. Somatostatin receptor scintigraphy before and after treatment with somatostatin analogues in patients with thyroid eye disease. Thyroid. 1999; 9(1): 47-52, doi: 10.1089/thy.1999.9.47, indexed in Pubmed: 10037076.

13. Burggasser G, Hurtl I, Hauff W, et al. Orbital scintigraphy with the somatostatin receptor tracer 99mTc-P829 in patients with Graves' disease. J Nucl Med. 2003; 44(10): 1547-1555, indexed in Pubmed: 14530465.

14. Durak H, Söylev M, Durak I, et al. Tc-99m polyclonal human immunoglobulin G imaging in Graves' ophthalmopathy. Clin Nucl Med. 2000; 25(9): 704-707, doi: 10.1097/00003072-200009000-00011, indexed in Pubmed: 10983759.

15. Galuska L, Leovey A, Szucs-Farkas Z, et al. SPECT using 99mTc-DTPA for the assessment of disease activity in Graves' ophthalmopathy: a comparison with the results from MRI. Nucl Med Commun. 2002; 23(12): 
1211-1216, doi: 10.1097/01.mnm.0000046214.83338.50, indexed in Pubmed: 12464787.

16. Galuska L, Varga J, Szucs Farkas Z, et al. Active retrobulbar inflammation in Graves' ophthalmopathy visualized by Tc-99m DTPA SPECT. Clin Nucl Med. 2003; 28(6): 515-516, doi: 10.1097/01.RLU.0000067520.87766.B6, indexed in Pubmed: 12917542.

17. Galuska L, Leovey A, Szucs-Farkas Z, et al. Imaging of disease activity in Graves' orbitopathy with different methods: comparison of (99m)Tc-DTPA and $(99 \mathrm{~m})$ Tc-depreotide single photon emission tomography, magnetic resonance imaging and clinical activity scores. Nucl Med Commun. 2005; 26(5): 407-414, indexed in Pubmed: 15838422.

18. Iyer S, Bahn R. Immunopathogenesis of Graves' ophthalmopathy: the role of the TSH receptor. Best Pract Res Clin Endocrinol Metab. 2012; 26(3): 281-289, doi: 10.1016/j.beem.2011.10.003, indexed in Pubmed: 22632365.

19. Ujhelyi B, Erdei A, Galuska L et al. Retrobulbar 99mTc-diethylenetriamine-pentaacetic-acid uptake may predict the effectiveness of immunosuppressive therapy in Graves' ophthalmopathy. Thyroid. 2009; 19(4): 375-380, doi: 10.1089/thy.2008.0298, indexed in Pubmed: 19355827.
20. Cooper IF, Siadaty MS. Quantitative Concepts' associated with '99m Technetium Diethylenetriaminepentaacetic Acid. Top Publications. BioMedLib Review. 2014; 11(9)

21. Galuska L, Varga J, Szücs-Farkas Z, et al. Differences in SPET analysis of thyroid-associated orbitopathy. Eur J Nucl Med Mol Imaging. 2004; 31(5): 793-795, doi: 10.1007/s00259-004-1459-3, indexed in Pubmed: 14767621.

22. Szabados L, Nagy EV, Ujhelyi B, et al. The impact of 99mTc-DTPA orbital SPECT in patient selection for external radiation therapy in Graves' ophthalmopathy. Nucl Med Commun. 2013; 34(2): 108-112, doi: 10.1097/MNM.0b013e32835c19f0, indexed in Pubmed: 23196678.

23. Hauser W, Atkins HL, Nelson KG, et al. Technetium-99m DTPA: a new radiopharmaceutical for brain and kidney scanning. Radiology. 1970; 94(3): 679-684, doi: 10.1148/94.3.679, indexed in Pubmed: 4984092.

24. Alevizaki-Harhalaki M, Alevizaki C, Georgiou E, et al. Increased Tc-99m DTPA uptake in active Graves' ophthalmopathy and pretibial myxoedema. J Nucl Med. 1983; 24(2): 174-176, indexed in Pubmed: 6337239. 